\title{
PARA LA FECHA DEL YEÍSMO Y DEL LLEÍSMO
}

Entre los varios artículos que escribió en los últimos años de su vida mi querido amigo y maestro don Amado Alonso, todos muy densos y trabajados, ninguno me parece tan nuevo e interesante como el que publicó sobre "La $l l$ y sus alteraciones en España y América" en los Estudios dedicados a Menéndez Pidal, volumen II, páginas 41-89. A la perspicuidad y la penetración que se hallan en todos sus escritos se agrega aquí una simplicidad en las afirmaciones que no hubieran permitido otros temas más oscuros e intrincados de que trató en otras partes, y al final nos regala con un panorama de la evolución general del consonantismo castellano desde fines de la Edad Media, trazado con mano segura y magistral. No podemos menos de notar ahí cosas tan nuevas como la afirmación implícita de que la $v$ era labiodental en aquella época (pág. 83), y la categórica y repetida de que la $j$ castellana medieval era africada y no fricativa (págs. 82 y 84). Con verdadera impaciencia hemos de esperar todos los dos tomos del tratado que dejó casi listo el gran desaparecido, donde estos juicios vendrán sin duda apoyados en un sólido conjunto de pruebas $^{1}$.

El resultado de mayor bulto en este trabajo es la fecha moderna del yeísmo en todas partes, posterior al siglo xvir en judeoespañol, no atestiguado hasta por los años de 1680 en América, sólo desde cien años más tarde en Andalucía y en fechas todavía más recientes en las demás regiones españolas que lo conocen; de ahí, pues, que no pueda ser importación andaluza en América ni en los Balcanes, sino innovación surgida espontáneamente en muchos focos autónomos. Los datos y razones en que se apoya la afirmación de estos hechos son firmes y sólidos. La ausencia de testimonios positivos en muchos

\footnotetext{
I Semanas antes de su muerte, tuvo don Amado la atención de remitirme la colección de esas tiradas aparte, avalorándola todavía con útiles correcciones que escribió al margen un amanuense, evidentemente por su encargo. Es importante entre ellas el detalle de que el padre Juan Villar, autor del Arte de gramática de $1_{51}$ (págs. 51-52), no residió en Granada, sino verdaderamente, como dice él mismo, "en está parte occidental de el Andaluzia", y concretamente en Sevilla, Utrera y Córdoba (supongo hay que leer así en vez de "Cardona"). Resulta pues de ahí que el yeísmo no sólo debía de ser desconocido por estas fechas en la provincia de Jaén, patria de Villar, sino también en el Occidente de Andalucía.
} 
gramáticos y fonetistas de los siglos xvi y xvir que notan otras igualaciones fonéticas de su época es ya convincente. Sin duda es verdad, como escribió el propio Alonso, que después de la primera mitad del siglo xvi los gramáticos se copian y repiten en forma tan mecánica como poco inteligente; luego es lícito todavía dudar del valor de esta deducción ex silentio en cuanto a los posteriores a 1650 . Pero si alguien quisiera llevar mucho más allạ su escepticismo, quedaría siempre el argumento sin réplica: si los moriscos decían zebolia o cabalio es porque nadie pronunciaba todavía ceboya ni ca. bayo; de lo contrario habrían adoptado estas pronunciaciones, puesto que la y era fonema corriente en árabe.

En lo referente a América abundaré en el sentido de que no se hallan datos anteriores a fines del siglo xvir. La experiencia que tuvo Alonso con una grafía de 1593 , falsa y sin fundamento, la repetí yo en varios casos. En la edición que dió Márquez Miranda de la Doctrina christiana y cathezismo en lengua millcayac, publicada por el padre Valdivia en Lima en 1607 , se lee iantico en la traducción del millcayac nac nac, y el editor lo interpreta como un diminutivo de llanto; pero el facsímil de la edición príncipe que acompaña la de Márquez nos permite leer iuntico, de acuerdo con el orden alfabético, y entender 'junto, cerca', de conformidad con el sentido de 'corta distancia' que había de tener aquella voz indígena, en vista del allentiac nac 'corto'. El nombre del cacique guarpe del siglo xvi, Guaimallén, hoy conservado como denominación de un arrabal de Mendoza, debe de contener un caso de $l l$ por $y$, pues, según indicó Canals Frau, puede analizarse en la lengua indígena como Guaima$y e$, pero de hecho la grafía con $l l$ parece ser moderna, pues en la única mención coetánea que tengo anotada, de 1613 , aparece escrito Guaimayé (AIEA, V, 1944, pág. 106, nota). Más antigua puede ser la $y$ por $l l$ en el nombre de otro cacique mendocino, Allaime, hoy conservado como nombre de un valle de la Precordillera y de un pueblo del Noreste de la provincia: en varios documentos de la época lo encontramos ya así, mas en otros $(1564,1590,1613)$ aparece Allal$m e$, y es de creer que se trate de un derivado del millcayac allall 'oro' (ibid., pág. 126, nota), pero aquí estamos en un caso especialísimo, el de 1 ante consonante, especie desusada en romance y repugnante a la articulación castellana, que es natural sustituyera esta 1 , imposible en su sistema, por el sonido vecino $\mathbf{y}^{2}$.

En cuanto a España, es convincente la idea de Alonso de que el

"Sustitución que se practica en otros indigenismos: payca 'bifurcación' $<$ quechua pallca (LAFone Quevedo, Tesoro de catamarqueñismos), Indapaico población de la provincia de San Juan. El mismo idioma millcayac, que poseía los dos sonidos de 1 y de $y$, conservaba como 1 la $l l$ castellana de los préstamos (por ejemplo llahuy 'llave' en el citado vocabulario del padre Valdivia), prueba de que no sonaba como $y$. 
yeísmo ha aparecido y se ha propagado independientemente desde varios focos. Y no sólo aseguraría que hubo más que los señalados hasta ahora, sino que falta en la lista el más curioso de todos, por ser el más antiguo y por haberse producido en una zona donde hoy reina la 1 en forma compacta, la aragonesa y la parte oriental del territorio de lengua castellana. (Véase el mapa). Pero lo más extraño es que los hallados en esta zona son casos de lleísmo, no de yeísmo, y numerosos por cierto.

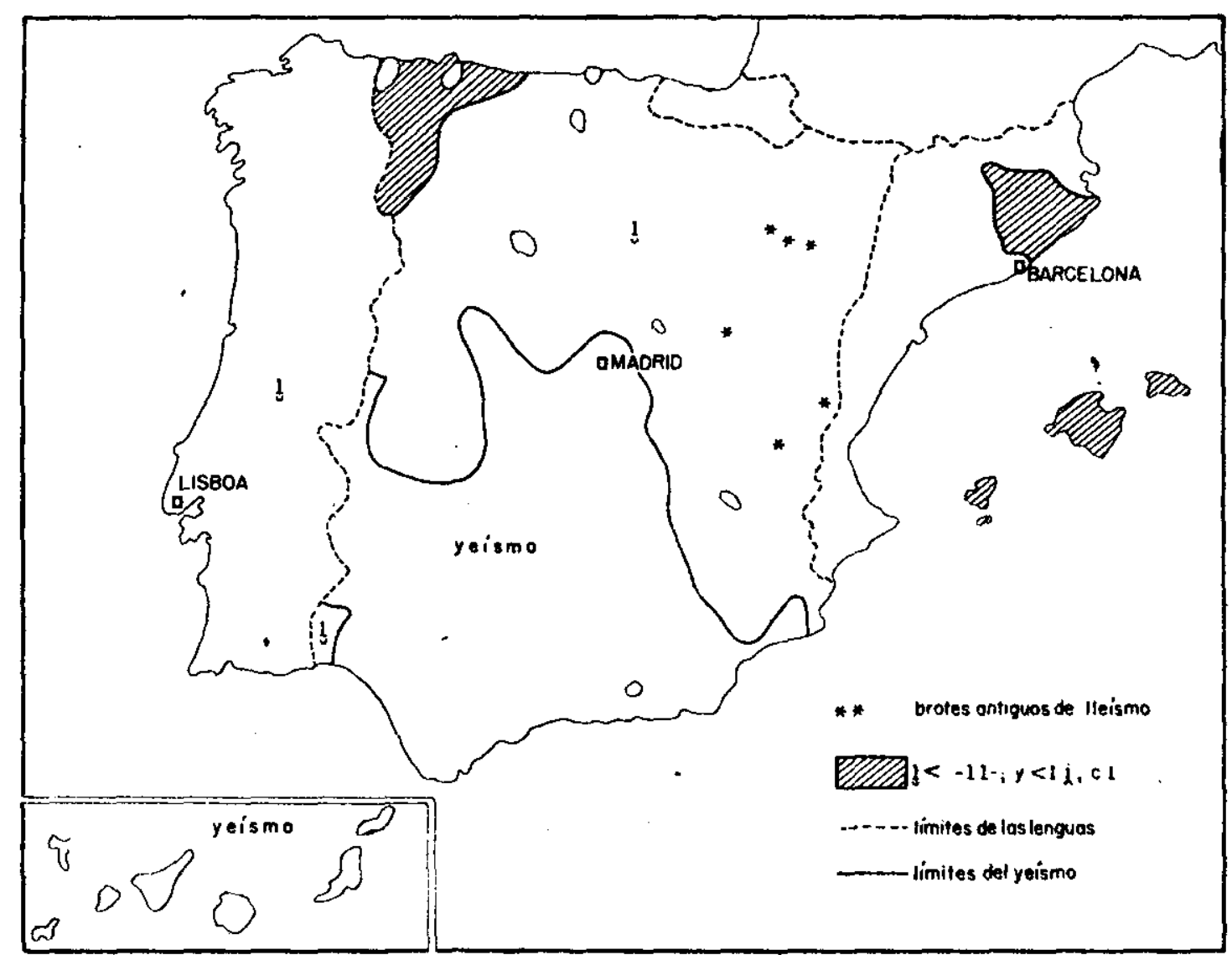

En el manuscrito $\mathrm{P}$ del Libro de Alexandre, escrito en Aragón en el siglo xv, recuerdo haber anotado varios; ahora sólo encuentro uno, pero éste es clarísimo (copla $\left.3{ }^{1} 5 a\right)$ :

Archiles so, que llago, so este mármol, çerrado, el que ovo a Étor, el troyano, rrancado; matóme por la planta Paris el perjurado,

donde el manuscrito $O$ trae iago, del verbo yacer, según es evidente. El fenómeno abunda mucho en el glosario del Escorial, también aragonés y escrito hacia 1400: "llelo: gelu", "llema de huevo: vitellus", "callado: agulus" (cf. agolus traduciendo a cayada en el glosario de Toledo), "aollar: pastino" (sin duda derivado de hoyo, pues pastino valía 'trabajar con la azada'), "papagallo: citacus" (es decir psittacus). $Y$ lo vemos repetirse en fuentes muy diversas, pero todas correspondientes al Este del territorio lingüístico: "Un capisayllo pardo", por 'capisayo', en inventario de Zaragoza, año 1402 (BAE, III, 1916, pág. 360). La planta gayuba 'Arctostaphyllos uva-ursi', cuyo nombre por lo 
demás aparece siempre con $-y$ - desde la Edad Media, y parece ser un prerromano * a g a ú a, que con metátesis dió en Francia * a ja ú ga, *a já ug a (gasc. yaugue, fr. dial. ajou, fr. ajonc: $R E W, 4579$ ), se llama hoy galluva en Utiel (zona aragonesa de Valencia), gallubera en Titaguas (id. de Castellón), galluvera en la provincia de Guadalajara, de donde el préstamo valenciano gallufa, gallufera, y también, es verdad, el gall. agallúa $a^{3}$. Es aragonés moderno rallo 'alcarraza', seguramente derivado del verbo rayar 'correr (agua), manar', más conocido como cat. rajar $\mathrm{r}$ a diare. Y es netamente aragonés bodollo 'podón', ya documentado en 1625 , procedente de *vedoyo y éste del celto-latino vi d u bi u m.

Por lo demás, desde Aragón pudieron propagarse palabras de este tipo hasta hablas vecinas. El propio $v$ i d u b i u m aparece en la forma bedoulh extendido por casi toda Gascuña, en Arán, Comminges, Gers, Landas y Bearne, y desde Gascuña hubo de pasar al Angoumois, pues Antoine Thomas recogió tres ejemplos de bedouil o vedouil en tres documentos del siglo $\mathrm{xv}$, desde 1444, procedentes de la Charente-Inférieure ${ }^{4}$. Ahora bien, el carácter advenedizo del vocablo es indudable en este departamento, según muestran la $b$ - y la conservación de la -D-. En cuanto a Gascuña, quizá cupiera en rigor admitir un desarrollo autóctono, aunque relacionado con el aragonés. El propio Thomas llamó la atención hacia meroil (de $\mathrm{m}$ a rrubium) en el languedociano Daudè de Pradas (hacia 1225). El cast. arroyo aparece alterado en forma análoga en varios dialectos de la otra vertiente de los Pirineos occidentales: suletino y bajonavarro arroil 'reguera, atarjea', 'fosa', 'garganta entre montes', arroilha-estaila 'alcantarilla', bearn. arroulhe 'rigole, ornière, fossé, canal', Lescun arroulho 'petit fossé's. Finalmente, el cast. fayanca 'cosa de poco valor', 'postura falsa', 'engaño', en portugués faianca 'objeto grosero, mal hecho' (quizá conexos con los gall. fayado 'techo, desván', fayar 'poner el techo' y derivados como éstos de faia 'haya'), no relacionados en todo caso con fallar ni con f a ll e re, pues desde Lope, Tirso y Correas aparecen constantemente escritos con - $y$-, han pasado en fecha reciente al catalán en la forma fallanca, claro está que trasmitidos a través de Aragón.

El paso por esta región o por sus aledaños navarro-vascos será indudablemente la causa de que ciertas palabras galorrománicas hayan cambiado en $l l$ - castellana su primitiva $y$ - Así sobre todo llan-

${ }^{3}$ M. Colmeiro, Enumeración de las plantas de la Peninsula..., Madrid, I $885^{-1889}$, vol. III, pág. 523 .

${ }^{4}$ Essais de philologie française, pág. 254.

"Claro que es arbitrario postular un diminutivo *a rr ug u l u m (como quisiera J. Hubschmid, Troisième Congrès International de Toponymie, Louvain, 195I, pág. 1 85), pues no podría salir del conocido a r rugia. También ha habido quien forjara un * v i d u c u l u m. No deben aislarse estos casos uno del otro. 
$t a$, ya escrito en esa forma en 1599 (diccionario de Percivale), y procedente del fr. jante, celta ca $\mathrm{m}$ b i t a, por conducto del gasc. yante. Lleta 'tallo recién nacido', voz menos conocida y que no me consta dónde se emplee, debe de venir de su sinónimo francés jette (equivalente dialectal de rejeton), derivado de jeter, y sin duda también por intermedio de un gasc. yete; quizá lleta sea palabra navarro-aragonesa, pues aparece por primera vez en el vasco Terreros (hacia 1764), aunque no la creo autóctona en esta región, ya que en sílaba tónica esperaríamos * cheta como representante alto-aragonés de jectare (aunque en posición átona se encuentre itar procedente de este infinitivo).

Hay, finalmente, unos pocos casos del fenómeno que parecen ser autóctonos en Castilla, o por lo menos tienen arraigo allí. En el caso de trullo 'especie de pato que se sumerge', aunque ya admitido por el Dicc. Aut. sin nota regional, y documentado desde 1640 en Martínez de Espinar, todavía cabe que sea voz principalmente aragonesa; en todo caso apenas cabe dudar de la etimología t $r$ u o, que en latín vale lo mismo, y pasaría primero por *truyo, como suyo y tuyo de s u u s, t u u s. Pero de uso general son desde antiguo grulla (de g r u e m), ya medieval junto al antiguo grúa, y pulla, que sale primeramente en Nebrija, y cuya identidad con puia o puya en el sentido de 'expresión hiriente' parece bien establecida por los datos que he reunido en mi diccionario ${ }^{6}$. Tal vez en estos casos, dada su antigüedad y generalidad en Castilla, deberemos buscar una influencia leonesa, de la zona centro-occidental, donde formas como muller y fillo estaban en lucha con muyer, fiyo y análogas, y con ella pudo confluir una corriente aragonesa desde el otro lado.

Lo que más sorprende es que todos los casos, y tantos en número, sean de lleísmo y no de yeísmo. Pues es difícil concebir lo uno sin lo otro. Los numerosos ejemplos de $l l$ por $y$ reunidos por Alonso (págs. 77-79) y los muchísimos modernos que podríamos agregar, o son meras grafías inversas, o a lo sumo ultracorrecciones fónicas, mạ́s o menos ocasionales, de la pronunciación de $l l$ como $y$, debidas a elementos sociales que tratan en vano de conservar la distinción tradicional. En la masa de datos medievales y aragoneses que he reunido no cabe dudar, en cambio, de que se trata de pronunciaciones,

${ }^{6}$ Desde luego hay que desechar la base diminutiva * gr u i l I a, supuesta por algunos, que no tendría justificación semántica en una ave tan grande y que no despierta sentimientos afectivos; el resultado, además, sólo podía ser * gruella o * gruílla, que no tenía por qué trasladar el acento, cuando justamente el castellano cambia $u i$ en $u i$. No menos falsas son las etimologías s u ill u s >chulla, chuleta (que en realidad es préstamo del cat. xulla $<$ anxunya $<$ cat. ant. ansunya a xungia), y zolle, que no viene de suĩle, sino del vasco. La etimología p ŭ l I a 'especie de higo' no puede aceptarse para pulla fonética ni semánticamente. 
y más o menos permanentes. $\mathrm{Y}$ asombra no hallar otros tantos ejemplos del fenómeno opuesto, pues si el sonido de 1 por su índole compleja es poco estable y ha tendido a convertirse en y en muchas épocas e idiomas diversos, no sería fácil comprender en virtud de qué tendencia podría una y cambiarse espontáneamente en el fonema complejo $1^{7}$. Quizá sea casual la preponderancia del lleísmo, pues al fin y al cabo no faltan del todo algunos casos aragoneses y antiguos de y por 1 . Uno hay en el códice aragonés del Alexandre (copla $677 c)$ :

vayámoslo ferir, nol tengamos belmez:

sy él me acomete, él se yeva [O: levará] el prez, ternién todos que fuy de coraçón rrafez.

Y el navarro y aragonés trias 'carriles' me parece claro que es derivado de trillar, camino trillado, pues no cabe mirarlo como variante del latinismo estrias, cuyo significado es demasiado distinto.

Siendo así, lo natural es pensar que hubo un brote de yeísmo en las zonas de Aragón y vecinas en los últimos siglos de la Edad Media, tendencia que no llegó a consolidarse; una reacción enérgica la haría abortar, y como suele acaecer en tales casos se han conservado más recuerdos de la reacción exagerada que de la tendencia misma (cf. en francés el caso secundario de chair y de berge, tanto o más frecuente que el primitivo de harde y larme, o el de chaise y besicles, decididamente más extendido que el del rotacismo primitivo). Es más probable que el foco estuviese en la llanura central aragonesa o en el Sur de Aragón que en la zona pirenaica, pues de allí es de donde vienen los pocos casos bien localizados (Zaragoza, Titaguas, Utiel, Guadalajara). El caso es que hoy el grupo navarro-aragonés y el castellano oriental es el que en forma más compacta permanece fiel a la l. (Véase el mapa).

$\mathrm{Y}$ en las hablas alto-aragonesas, las conservadas en forma más fiel a su punto de partida medieval, no hay casos de yeísmo. Desecho desde luego la posibilidad, tomada en cuenta por Alonso (pág. 59), de que el cambio de LL en $c h$ (griĉones, saldjéĉo, po $\theta$ jéĉo), atestiguado en Echo, Araguiés y otros pueblos del Noroeste aragonés, pueda explicarse por africación de una y más antigua. En otra zona más oriental, valles de Broto y de Tena, encontramos el resultado $\mathrm{t}$ : castieto, vertubieto ( $\mathrm{v}$ e $\mathrm{r} \mathrm{t} \mathrm{b}$ e $11 \mathrm{um}$ ). Y es evidente que estamos ante una prolongación del área gascona donde -LL' va a parar en unas partes a $-\hat{\mathbf{c}}$, en otras a $-\mathbf{t}$, y en otras se conserva en la forma $-\mathbf{t}$

'Pueden dejarse aparte un par de casos catalanes de $y$ - $>l l$ en posición inicial: el mall. Lloatxim 'Joaquín' y el común llessamin junto a gessami 'jazmin'. La posición de la consonante facilita ahí la acción de la $l$ del artículo, y en el segundo caso la amalgama pudo ya producirse con el artículo árabe (al-yāsamîn). 
(escrito ordinariamente -th), que ha de ser lo más antiguo: castetch, castet o casteth; betetch, betet o beteth ( $\mathrm{v}$ i t e $1 \mathrm{lu} \mathrm{m})$. Aun en los valles, como el de Arán, que hoy dicen pet pell is, saumet, capet, aquet (e c c u m i $11 \mathrm{um}$ ) y análogos, hay en cambio -tch tras o y u: motch mollis, poutch pullus, bedoutch, arrastoutch, etc., lo que prueba que empezó por haber palatalidad tras todas las vocales, elemento luego reabsorbido por una vocal palatal antecedente. Es probable que exista una relación entre todo esto y el tratamiento ḍ de la LL en Cerdeña y Sur de Italia, pues al fin y al cabo palatal y cacuminal son siempre articulaciones más traseras que la de la $\mathrm{L}$. Por la misma razón dudo de que los casos asturianos de gatso, vatse, tsobu deban explicarse por un yeísmo anterior. Téngase bien presente la demostración concluyente que nos ha dado Alonso de que la $l l$ pasó directamente a ž en el Ecuador y en el valle de Orizaba, lo mismo que debió de ocurrir en la Castilla medieval, pues de haber habido una etapa intermedia y, las voces del tipo de mayo se hubieran mezclado con las del de ajo. Análogamente, una 1 de intensión más enérgica podía pasar directamente a una africada $\breve{g}$, de donde los resultados posteriores $\hat{\mathbf{c}}, \hat{\mathbf{s}}$, etc. ${ }^{8}$

Juan Corominas

The University of Chicago.

Institut d'Estudis Catalans.

8 A la discusión de las págs. 88-89 con Zamora Vicente será oportuno aducir mi testimonio de catalán que vivió seis años en la Argentina, y distingue los

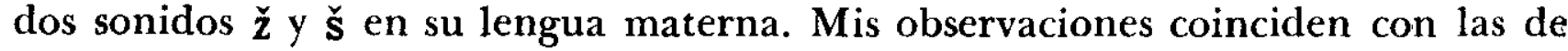
Alonso. La pronunciación š de la $l l$ se oía incomparablemente menos que ž en los años 1939-1945. Tampoco creo que atine Zamora al decir que aquélla es propia de las clases trabajadoras y semicultas y ésta de la gente educada. Aunque en Mendoza se pronuncia $\mathbf{y}$, oí a algunos centenares de porteños que 'pasaron por Mendoza, yo mismo viví cerca de un año en la capital, y sólo recuerdo dos casos de personas que pronunciaban š, por lo demás en forma constante: ambas eran personas jóvenes de familia acomodada. En otros se oía con carácter más ocasional, casi siempre en gente joven. Parecía tratarse de una pronunciación en avance, pero muy minoritaria todavía, y más bien normal o descuidada que enfática. 